\title{
Artificial Intelligence Based State Observer in Polymerization Process
}

\section{Jarinah Mohd Ali ${ }^{1}$}

M.A. Hussain *,1

${ }^{1}$ Department of Chemical Engineering, Faculty of Engineering University of Malaya, 50603 Kuala Lumpur

`e-mail : mohd_azlan@um.edu.my

Observers or state estimators are devices used to estimate immeasurable key parameters that are due to noise, disturbances and mismatch. It is important to identify those variables prior to construct a control system and avoid fault or process disruption. In certain chemical processes, such observer usage produced unsatisfactory results therefore hybrid approached is the appropriate solution. Hybrid observers are combination of two or more conventional observers mainly to enhance the estimator's performance and overcoming their limitations. In advanced cases, Artificial Intelligence algorithm is applied. This paper develops two hybrid observers namely sliding mode and extended Luenberger observers with fuzzy logic for approximating the monomer concentration in a polymerization reactor. It was found that the sliding mode observerfuzzy combination is better based on noise handling with less oscillation.

Keywords : Artificial Intelligence, Fuzzy logic, State estimation, Polymerization, Reactor

\section{INTRODUCTION}

Monomer concentration in polymerization reactor is one of the favorable parameters that will affect the product quality if it is not control. Several researchers have made a good effort in estimating the concentration prior to implement the control procedure in a polymerization reactor using a device called observer or state estimator (Vicente et al. 2000) (Zambare et al. 2002) (Wei et al. 2007) (Ng \& Hussain 2004). Observers or state estimator will approximate unknown variables with simple formulation and high rate of convergence to reduce the usage of expensive sensors. They have applied many types of observers including receding horizon estimator (BenAmor et al. 2004), Extended Kalman Filter (EKF) (Gentric et al. 1999) and open loop observer (Vicente et al. 2000).

Extended Luenberger observer (ELO) and sliding mode observer (SMO) are two types of conventional observers that are related to each other where SMO is the extended version of ELO. ELO has once been applied to estimate the monomer concentration but only based on the nonobservable parameters (BenAmor et al. 2004) while SMO has been merged before with proportional observer (Aguilar-López \& Martinez-Guerra 2005) for similar purpose. In some cases, the conventional 


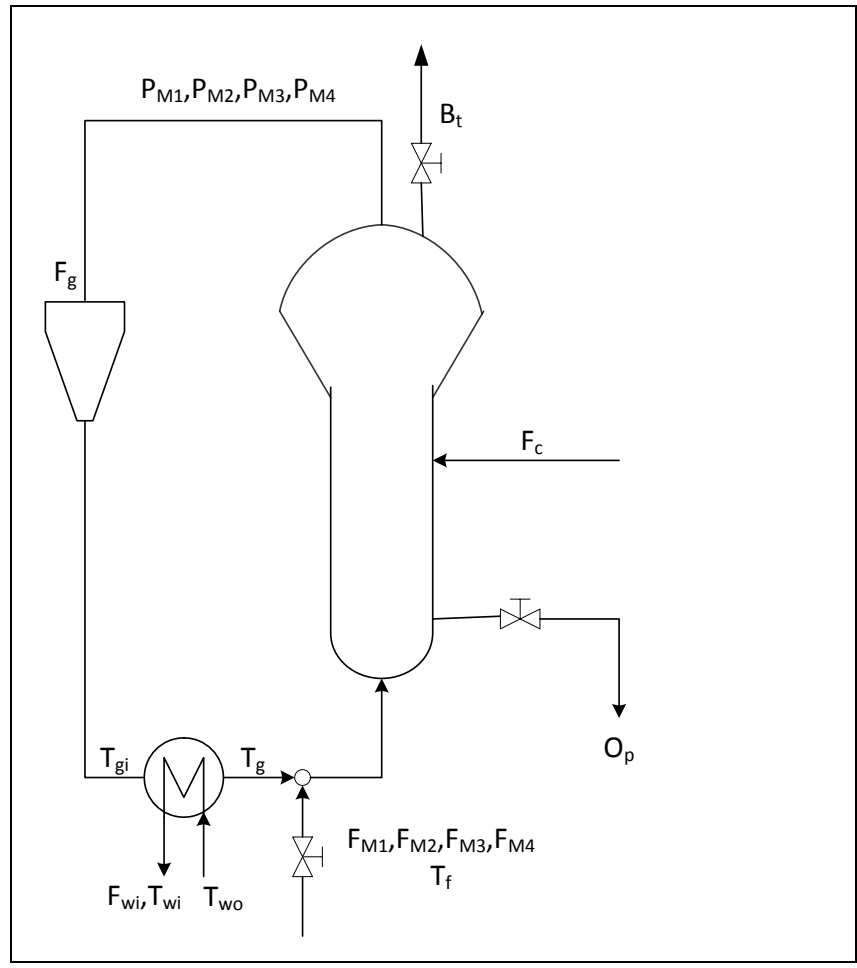

Fig. 1: Polymerization reactor for producing polyethylene min residence time

observer alone will result in unsatisfactory outcome such as offsets and the best option to avoid this is combining it with artificial intelligence (AI). Fuzzy logic is the simplest algorithm of $\mathrm{AI}$ and is suitable to be merged with those conventional observers based only on the IF and THEN rules which can be manipulated to obtain better estimation performances.

Thus, this paper develops two hybrid observers to predict the monomer concentration in order to overcome the limitations of single observer as well as provide the simplest way in designing a hybrid estimator by applying fuzzy rules only in the estimation error, and the change of error to obtain desired results with fast and less efforts. Introduction is in this section follow by the polymerization process in section 2. The observer design based on a case study is discussed in section 3 while future work and conclusion are in section 4.

\section{POLYMERIZATION PROCESS}

The polymerization process applied here is based on the well-mixed UNIPOL model of polymerization process developed by McAuley (1990) (McAuley et al. 1990; McAuley et al. 1994).The reactor used for producing polyethylene is illustrated in Figure 1 . The feed gas is merged with the recycled gas and enters the fluidized bed reactor while another part of fresh feed gas contains the Ziegler-Natta catalyst. Four major components entering the reactor are the monomer (ethylene), co-monomer (butane), hydrogen ( $\mathrm{H} 2)$ and nitrogen (N2). Nitrogen will carry the catalyst powder and maintain the column pressure at its desired value. The temperature of the reactor, on 
the other hand, is controlled by manipulating the feed temperature and cooling water.

By taking $M_{1}$ as ethylene, $M_{2}$ as butane, $M_{3}$ as hydrogen and $M_{4}$ as nitrogen, the process model is as follows:

$$
\begin{aligned}
V_{g} \frac{d C_{M_{1}}}{d t} & =F_{M_{1}}-x_{M_{1}} B_{t}-R_{M_{1}} \\
V_{g} \frac{d C_{M_{2}}}{d t} & =F_{M_{2}}-x_{M_{2}} B_{t}-R_{M_{2}} \\
V_{g} \frac{d C_{M_{3}}}{d t} & =F_{M_{3}}-x_{M_{3}} B_{t}-R \\
V_{g} \frac{d C_{M_{4}}}{d t} & =F_{M_{4}}-x_{M_{4}} B_{t}
\end{aligned}
$$

With $R_{M_{1}}=C_{M_{1}} Y_{c} k_{p 1}^{e^{\frac{E}{R}\left(1 / T-1 / T_{r e f}\right)}}$

$$
R_{M_{2}}=C_{M_{2}} Y_{c} k_{p 2}^{e^{\frac{E}{R}\left(1 / T-1 / T_{r e f}\right)}}
$$

For monomer concentration, the measured variable is given by:

$$
\frac{d Y_{c}}{d t}=F_{c} a_{c}-k_{d} Y_{c}-O_{p} Y_{c} / B_{w}
$$

Then, the error is as follows:

$$
e=C_{M_{1}}-\hat{C}_{M_{1}}
$$

With the error dynamic given by:

$$
\frac{d e}{d t}=\frac{d C_{M_{1}}}{d t}-\frac{d \hat{C}_{M_{1}}}{d t}
$$

Then including the error dynamic, observer's structure will take the form:

$$
\begin{aligned}
\frac{d \hat{C}_{M_{1}}}{d t}= & \left(F_{M_{1}}-\hat{C}_{M_{1}} B_{t}-R_{M_{1}}\right) / V_{g} \\
& +K \hat{C}_{M_{1}}\left(Y_{c}-\hat{Y}_{c}\right)
\end{aligned}
$$

$\frac{d \hat{Y}_{c}}{d t}=F_{c} a_{c}-k_{d} \hat{Y}_{c}-O_{p} \hat{Y}_{c} / B_{w}$

With $K$ as the observer's gain:

$$
K=\lambda_{1}-\lambda_{2}-2 \frac{B_{t}}{V_{g}}
$$

For a fast dynamic process $\lambda_{1}=-2$ and $\lambda_{2}=-2$

\section{OBSERVER DESIGN BASED ON A CASE STUDY}

ELO is first developed and is applied to estimate the monomer concentration and later SMO is designed to improve the estimation performances or eliminating the offsets. The basic equation of ELO is given in Eq. (13) while SMO is in Eq. (14).

$$
\begin{aligned}
\dot{x}(t)= & A \hat{x}(t)+B u(t)+K(y(t) \\
& -C \hat{x}(t)) \\
\dot{x}(t)= & A \hat{x}(t)+B u(t) \\
& +K \operatorname{sgn}(y(t)-C \hat{x}(t))
\end{aligned}
$$

$$
\begin{gathered}
\text { Where } \operatorname{sgn}(y(t)-C \hat{x}(t))= \\
\operatorname{col}\left(\operatorname{sgn}\left((y(t)-C \hat{x}(t))_{1}\right), \ldots, \operatorname{sgn}((y(t)-\right.
\end{gathered}
$$
$\left.C \hat{x}(t))_{n}\right)$

ELO for the monomer concentration based on measured co-monomer is given in Eq. (15) based on simplification of both Eq. (9) and Eq. (10). 


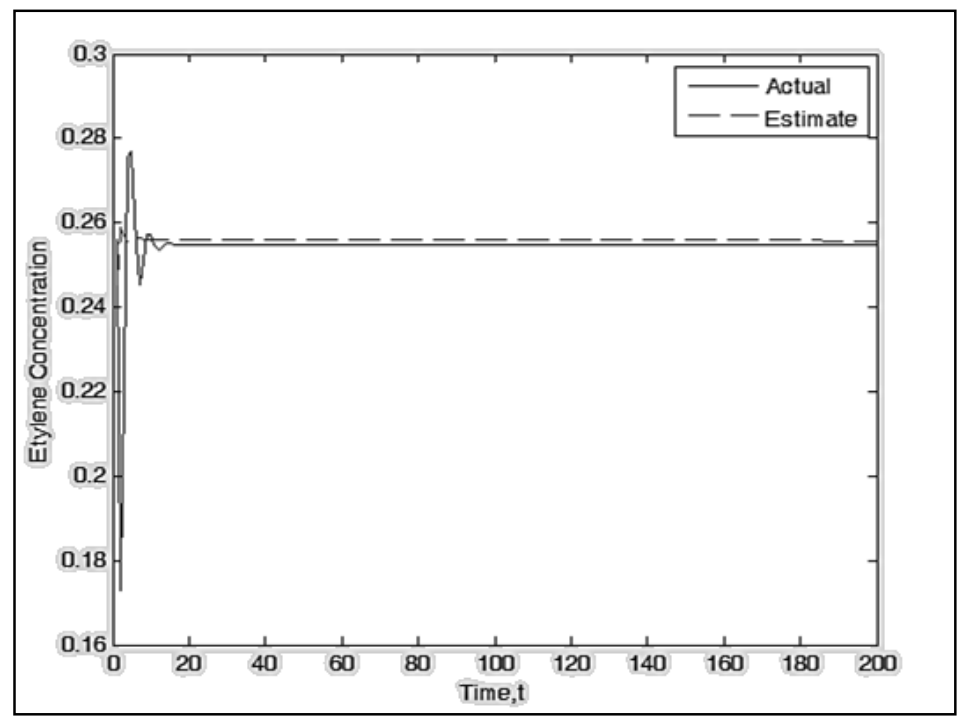

Fig. 2: Monomer concentration using ELO based on co-monomer concentration

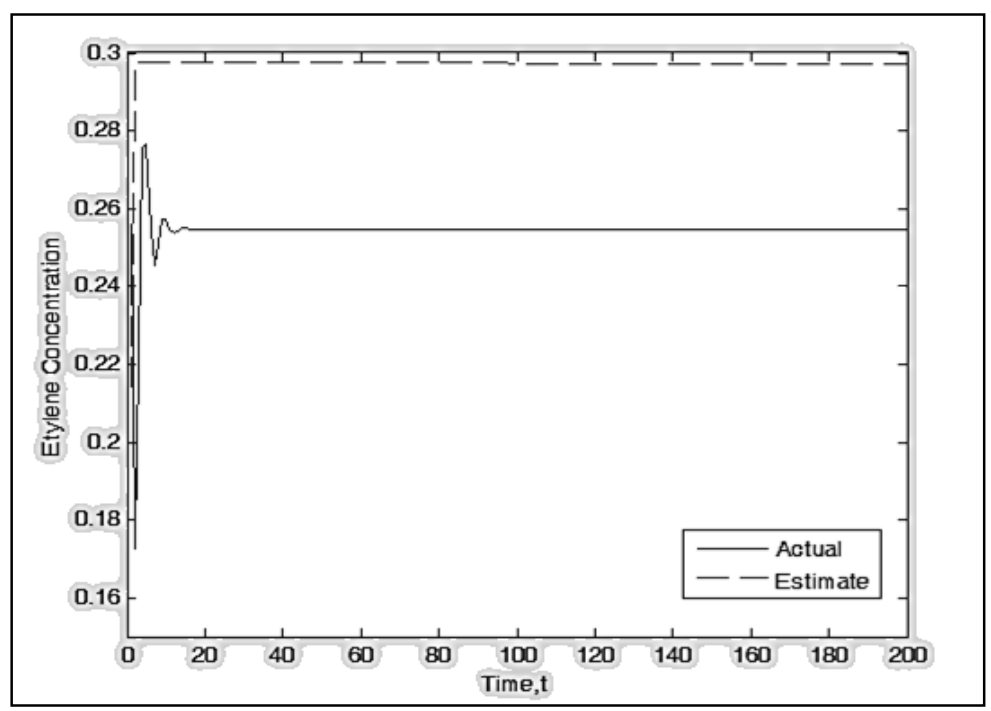

Fig. 3: Monomer concentration using SMO based on co-monomer concentration

ELO based on co-monomer concentration:

$$
\hat{C}_{M_{1}}=\frac{B_{t}}{V_{g}}\left(\hat{C}_{M_{1}}-C_{M_{1}}\right)+K\left(\hat{C}_{M_{2}}-C_{M_{2}}\right)
$$

By using simulink in MATLAB 2009, the observer is test compared to an actual model value of concentration and the results are illustrated in Figure 2. It showed non-convergence and with offsets as well as oscillation in the beginning thus another conventional observer, SMO is developed based on Eq. (16).

SMO based on co-monomer concentration:

$$
\hat{C}_{M_{1}}=\frac{B_{t}}{V_{g}}\left(\hat{C}_{M_{1}}-C_{M_{1}}\right)+K \operatorname{sgn}\left(\hat{C}_{M_{2}}-C_{M_{2}}\right)
$$

The method is repeated and the results are illustrated in Figure 3. ELO provide better results that reduce the offsets if compared to SMO; however it is still not 
converged. Hence another method will has to be designed and the best approached will be to merge those conventional observer with AI that will help in eliminating the offsets living convergence and better estimation performance. Fuzzy logic is chosen here since it can be combined using the IF, THEN rules by manipulating the error of estimation from the results of the conventional observers above. It is simpler since we already have the previous estimation results rather than designing another combination from scratch. The fuzzy rules are tabulated in Table 1.

Table 1. Fuzzy rules for concentration estimation

\begin{tabular}{cccccc}
\hline \multirow{2}{*}{$e$} & \multicolumn{5}{c}{$\Delta \mathrm{e}$} \\
\cline { 2 - 6 } & $\mathrm{NS}$ & $\mathrm{NL}$ & $\mathrm{ZO}$ & $\mathrm{PS}$ & $\mathrm{PL}$ \\
\hline $\mathrm{NS}$ & $\mathrm{PL}$ & $\mathrm{PL}$ & $\mathrm{ZO}$ & $\mathrm{ZO}$ & $\mathrm{ZO}$ \\
\hline $\mathrm{NL}$ & $\mathrm{PL}$ & $\mathrm{PL}$ & $\mathrm{ZO}$ & $\mathrm{ZO}$ & $\mathrm{ZO}$ \\
\hline $\mathrm{ZO}$ & $\mathrm{PS}$ & $\mathrm{PS}$ & $\mathrm{ZO}$ & $\mathrm{ZO}$ & $\mathrm{ZO}$ \\
\hline $\mathrm{PS}$ & $\mathrm{ZO}$ & $\mathrm{ZO}$ & $\mathrm{NS}$ & $\mathrm{NL}$ & $\mathrm{NL}$ \\
\hline $\mathrm{PL}$ & $\mathrm{ZO}$ & $\mathrm{ZO}$ & $\mathrm{NS}$ & $\mathrm{NL}$ & $\mathrm{NL}$ \\
\hline
\end{tabular}

The best fuzzy rules are obtained based on trial and error and the rules given above are manipulated three times to get accurate results. The best rule is then combined with both ELO and SMO to estimate the concentration. All results are given in Figure 4 and 5. From those figures, it is clear that Fuzzy-SMO is a better observer for estimating the monomer concentration as it provides fast convergence with less oscillation and able to handle noise if compared with the FuzzyELO. In addition, the co-monomer is the best measured variables that can be used in order to predict the concentration of monomer in a polymerization reactor.

\section{FUTURE WORK AND CONCLUSION}

Fuzzy-SMO is the best approached in predicting the monomer concentration in a polymerization reactor that able to handle noise and provide fast convergence with less oscillation compared to Fuzzy-ELO.

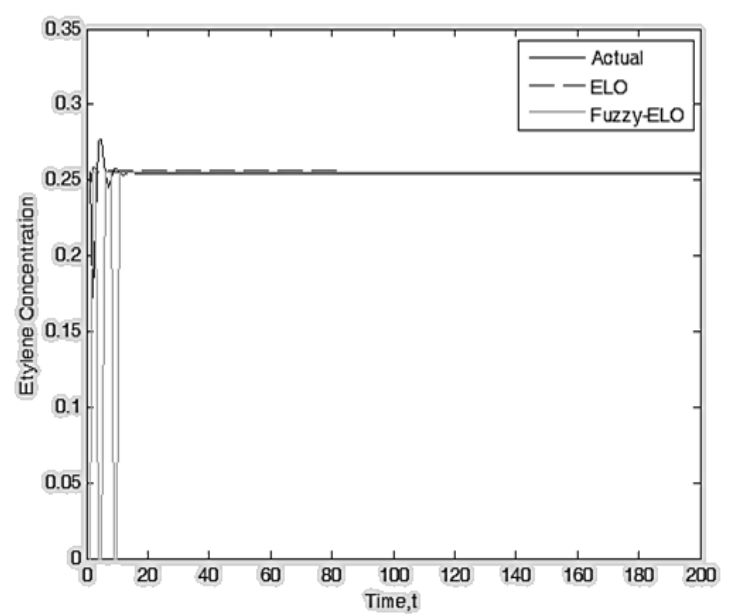

a) Without Noise

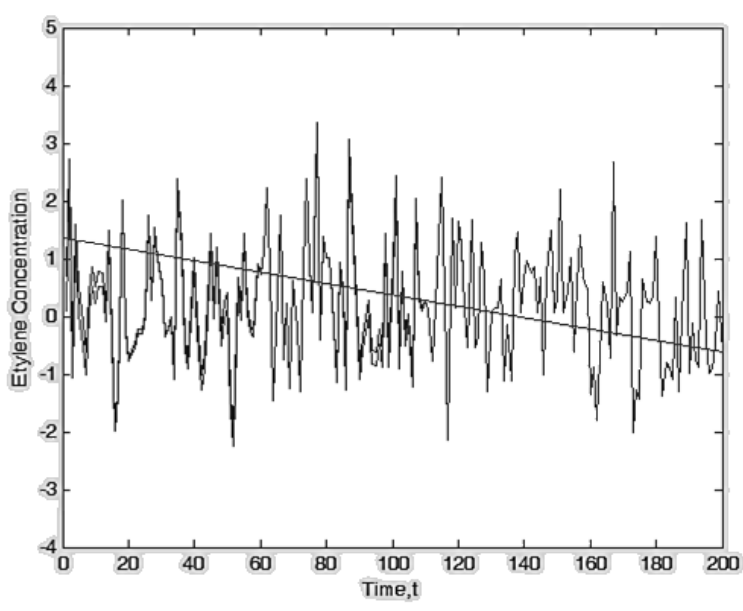

b) With noise

Fig. 4: Monomer concentration using Fuzzy-ELO based on co-monomer concentration 


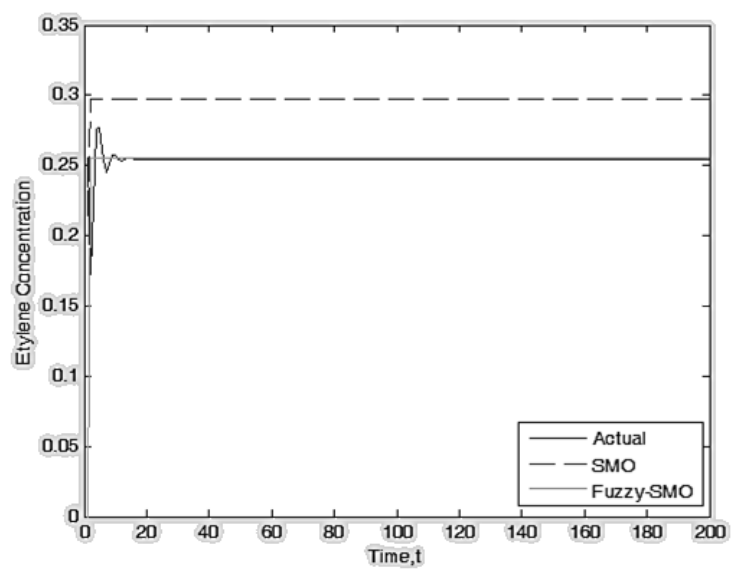

a) Without Noise

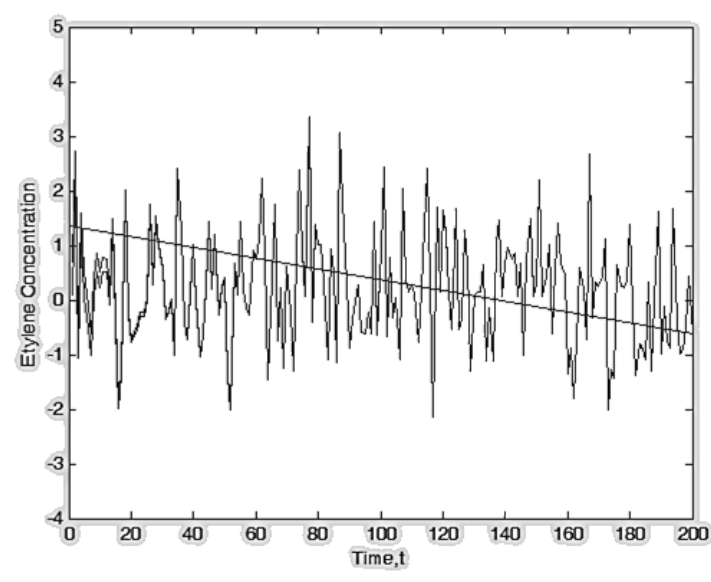

b) With noise

Fig. 5: Monomer concentration using SMO based on co-monomer concentration

Besides that, the most appropriate measured variable is the concentration of co-monomer itself where the relationship between those is the strongest and will result in higher conversion rate in contrast of using hydrogen, nitrogen and temperature. In future, the observer will be applied to predict several other key features in a polymerization process such as melt index, heat transfer coefficient, molecular weight distribution (MWD) and reaction rate using the hybrid observer.

\section{REFERENCES}

1. Aguilar-López, R., \& Martinez-Guerra, R. (2005). State estimation for nonlinear systems under model unobservable uncertainties: application to continuous reactor. Chemical Engineering Journal, 108(1-2), 139-144.

2. BenAmor, S., Doyle Iii, F. J., \& McFarlane, R. (2004). Polymer grade transition control using advanced realtime optimization software. Journal of Process Control, 14(4), 349-364.
3. Gentric, C., Pla, F., Latifi, M. A., \& Corriou, J. P. (1999). Optimization and non-linear control of a batch emulsion polymerization reactor. Chemical Engineering Journal, 75(1), 31-46.

4. McAuley, K., MacGregor, J., \& Hamielec, A. E. (1990). A kinetic model for industrial gas-phase ethylene copolymerization. AlChE Journal, 36(6), 837-850.

5. McAuley, K., Talbot, J., \& Harris, T. (1994). A comparison of two-phase and well-mixed models for fluidized-bed polyethylene reactors. Chemical Engineering Science, 49(13), 2035-2045.

6. Ng, C. W., \& Hussain, M. A. (2004). Hybrid neural network-prior knowledge model in temperature control of a semi-batch polymerization process. Chemical Engineering and Processing: Process Intensification, 43(4) , 559-570.

7. Vicente, M., BenAmor, S., Gugliotta, L. M., Leiza, J. R., \& Asua, J. M. (2000). Control of Molecular Weight Distribution in Emulsion Polymerization 
Using On-Line Reaction Calorimetry. Industrial \& Engineering Chemistry Research, 40(1) , 218-227.

8. Wei, N. C., Hussain, M. A., \& Wahab, A. K. A. (2007). Control of a Batch Polymerization System Using Hybrid Neural Network - First Principle Model. The Canadian Journal of Chemical Engineering, 85(6) , 936-945.
9. Zambare, N., Soroush, M., \& Grady, M. C. (2002). Real-time multirate state estimation in a pilot-scale polymerization reactor. AIChE Journal, 48(5), 1022-1033. 